\title{
Operating characteristics of thermoacoustic compression based on alternating to direct gas flow conversion
}

\author{
Kai Wang, Daming Sun*, Ya Xu, Jiang Zou, Xiaobin Zhang, Limin Qiu \\ Institute of Refrigeration and Cryogenics, Zhejiang University, Hangzhou 310027, PR China
}

\begin{abstract}
A thermoacoustic compressor is capable of converting an alternating gas flow to a direct one with a large pumping rate on the basis of the pressure oscillation nature of thermoacoustic engines and the flow rectification effect of check valves. Theoretical calculations are first carried out to study the factors that affect the performance of the closed and open thermoacoustic compression systems. It is shown that the frequencies of directly connected thermoacoustic engines should avoid small integer multiple relationships to operate efficiently. Increasing the pressure amplitudes is beneficial for the pressure lift in a closed system as well as the pumping rate in an open system. A demonstrative closed thermoacoustic compressor was then experimentally studied. A maximum average gas pumping rate of $4.55 \mathrm{Nm}^{3} / \mathrm{h}$ during the first $2 \mathrm{~s}$ of the compression process was achieved when all components were at the same initial mean pressure of $2.13 \mathrm{MPa}$. The maximum pressure lift reached $0.4 \mathrm{MPa}$ when the initial mean pressure was $2.4 \mathrm{MPa}$. It was found that the pressure lifts were roughly proportional to the pressure amplitudes. Due to the superposition of alternating and direct gas flows, deformation of pressure waveforms which has a negative effect on the performance was observed.
\end{abstract}

Keywords: Thermoacoustic; Compression; Pump; Flow rectification; Check valve

\section{Introduction}

Both alternating and direct flows are widely used in industries, such as in reciprocating and centrifugal compressors. In some applications, the two flow types even coexist. Taking G-M cryocooler [1] as an example, the gas flow supplied by the scroll helium compressor is direct flow while what required by the cold head is an alternating one. The rotary valve between the helium compressor and the cold head converts the direct gas flow into an alternating one by periodically switching between the high and low pressure ports of the compressor.

In recent years, the technology of flow type conversion has given birth to some novel promising applications. Recently, a mean flow acoustic engine composed of a main pipe and two single end closed branch tubes was proposed to induce acoustic oscillation from the mean flow (direct flow) in the main pipe [2-6]. Based on this conversion technique, aerodynamically driven thermoacoustic refrigerators and piezoelectric generators can be developed for mean flow energy (e.g. wind energy, fluid energy in pipelines) exploitation. With the development of smart materials, piezoelectrically driven hydraulic micropumps have been proposed for applications such as chemical process control, drug delivery system, and cooling of tiny electric element [7-9]. The hydraulic pump utilizes reed

\footnotetext{
* Corresponding authors. Tel/Fax: +86-571-87952769 (D. M. Sun)

E-mail address: sundaming@zju.edu.cn (D. M. Sun)
} 
valves, diffuser nozzles and active piezoelectric discs to convert the reciprocating motion of a piezoelectric actuator to a pumping action. In 2004, a resonant self-pumped circulating thermoacoustic heat exchanger was presented by Swift and Backhaus [10] to exchange heat with a remote heat source. Hydrodynamic asymmetrical constrictions were adopted as an imperfect gas diode to generate substantial mean flow out of a thermoacoustic engine. Later, check valves were adopted by Gao et al. [11] to realize the conversion from sinusoidally alternating to direct gas flow in a non-resonant self-circulating thermoacoustic heat exchanger.

Recently, we proposed a remarkable thermoacoustically driven compression effect based on the conversion of gas flow from an alternating state to a direct one [12-14]. Due to the unique features of thermoacoustic engines [15-18], thermoacoustic compression systems have the advantages of simple structure, thermally driven nature, oil-free operation and large operating range, and may find applications in gas compression, power generation, and refrigeration systems. If an expander is introduced further, a closed cycle solar power generation system or cooling system can be formed [14]. Besides, because of the already-demonstrated feasibility of micro-miniaturized thermoacoustic engines [19] and check valves [20-22], micro thermoacoustic compressors or pumps may find potential applications in MEMS field in the future.

In the previous paper [12], the thermoacoustic compression process is first proposed and a demonstrative thermoacoustic compressor verifies the feasibility of the concept. In order to reveal the operating principles and show the basic guidelines for the design of a thermoacoustic compression system, a detailed study of the working characteristics of closed and open thermoacoustic compression systems is carried out. The effects of operating frequency, pressure amplitude, phase difference, and mean pressure on the performance of such systems are presented in detail.

\section{Computation and analysis}

Fig. 1(a) shows the schematics of two types of thermoacoustic compression system, i.e., a closed and an open thermoacoustic compression systems. The closed thermoacoustic compression system (CTCS) consists of two or more thermoacoustic engines and in-between check valves, as denoted in the dashed box. The number of thermoacoustic engines is always one more than that of check valves in CTCS. The open thermoacoustic compression system (OTCS) consists of an arbitrary number of thermoacoustic engines and one more check valves. Unlike the CTCS inside which gas is always restricted, the OTCS continuously pumps gas from a low pressure gas source to a high pressure one. Check valves are set at the downstream and the upstream sides of the low and high pressure gas sources, respectively. CTCS can easily demonstrate the working principle of the thermoacoustic compression, while OTCS is more likely to be used in industry where continuous gas compression and pumping are required. The arrows in the figure show the directions of the gas flow. Due to the thermoacoustic conversion effect, the pressures $p_{H}$ and $p_{L}$ in the high mean pressure engine (HMPE) and the low mean pressure engine (LMPE) oscillate periodically with mean pressures $p_{H, m}$ and $p_{L, m}$, respectively, as shown in Fig. 1(b). As denoted by the shadows, the pressure $p_{L}$ in the LMPE can be higher than the pressure $p_{H}$ in the HMPE at some moments due to pressure oscillation. The check 


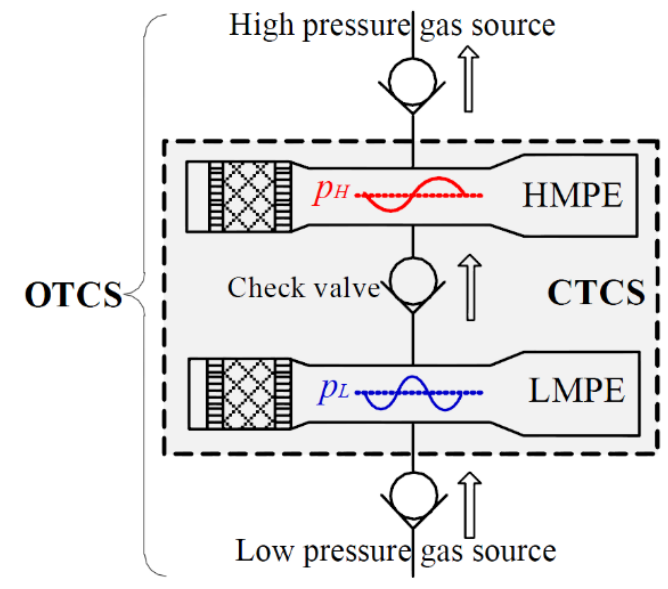

(a)

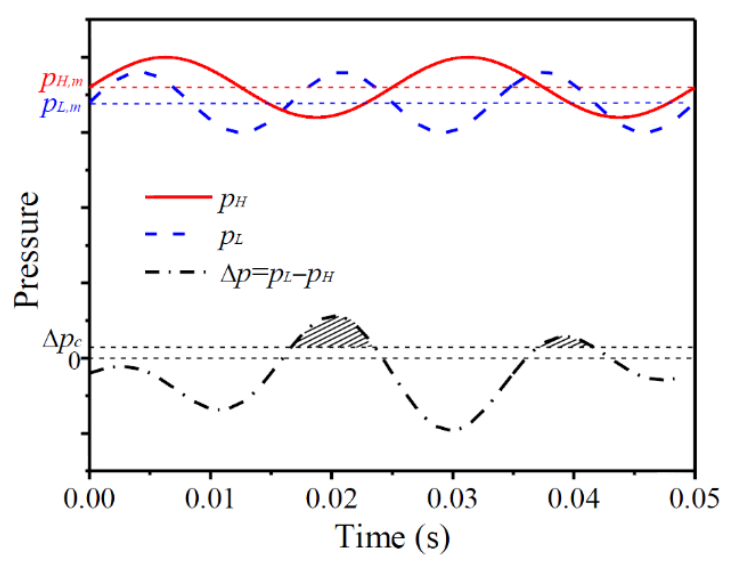

(b)

Fig. 1. Schematic for showing working principle of a thermoacoustic compressor.

valve enforces a one direction gas flow from the LMPE to the HMPE when the pressure difference $\Delta p=p_{L}-p_{H}$ exceeds the opening pressure difference $\Delta p_{c}$ of it. This is the main operating principle of a thermoacoustic compressor.

To simplify calculation, both the basic CTCS and OTCS units with nitrogen as working gas are studied. The basic CTCS unit consists of two thermoacoustic engines and a check valve. The volumes of the LMPE and the HMPE are 6.3 L and 5.3 L, respectively. The initial mean pressures of the LMPE and the HMPE are both set at $2.4 \mathrm{MPa}$ unless otherwise stated. Due to the thermoacoustic compression effect, the gas in the LMPE will be transported to the HMPE until the transient pressure difference $\Delta p$ across the check valve can not exceed $\Delta p_{c}$ anymore. The final pressure lift $\Delta p_{m}$ and pumping time are calculated. For the OTCS, as the gas is continuously supplied to the LMPE and discharged from the HMPE, the mean pressures stay constant. Thus, the pumping rate through the check valve is what applications concern. Therefore, a basic OTCS unit consisting of a check valve and two thermoacoustic engines at constant mean pressures is studied, and the average pumping rate through the check valve is calculated. The average pumping rates in both CTCS and OTCS in the computations are calculated via,

$$
U=\frac{\int_{0}^{\tau} \dot{U} d t}{\tau}=\frac{\int_{0}^{\tau} \frac{\dot{m} R_{g} T_{0}}{p_{0}} d t}{\tau}=\frac{m R_{g} T_{0}}{p_{0} \cdot \tau},
$$

where $R_{g}, T_{0}$, and $p_{0}$ are the gas constant, temperature, and pressure under standard conditions, respectively. $m$ and $\tau$ denote the total gas mass transported from the LMPE to the HMPE and the time needed to complete the compression process in CTCS. While in OTCS, $m$ denotes the total gas mass transported in the calculation time $\tau$, which is 10 seconds in the calculation. $\dot{m}$ and $\dot{U}$ denote the instantaneous mass flow rate and volumetric pumping rate through the check valve respectively.

The instantaneous mass flow rate $\dot{m}$ is determined by the pressure difference across the check valve $\Delta p$, and is calculated by [12,23]: 


$$
\dot{m}= \begin{cases}\sqrt{\Delta p \frac{2 \rho A^{2} R^{2}}{K F_{\text {mult }}}} & \text { if } 0<\Delta p<\Delta p_{c}, \text { fully closed } \\ \sqrt{\left(\Delta p-\Delta p_{c}\right) \frac{2 \rho A^{2}}{K F_{\text {mult }}}} & \text { if } \Delta p>\left(4 R^{2}+1\right) \Delta p_{c}, \text { fully open }, \\ \left(1+\frac{1}{4 R^{2}}\left(\frac{\Delta p}{\Delta p_{c}}-1\right)\right) \sqrt{\Delta p_{c} \frac{2 \rho A^{2} R^{2}}{K F_{\text {mult }}}} & \text { otherwise }\end{cases}
$$

where $\rho, A, K$ and $F_{\text {mult }}$ denote gas density, flow area of the check valve, local loss coefficient and empirical multiplier for viscous pressure drop, respectively. $R$, generally a positive number $<<1.0$, is the ratio of fully-closed to fully-opened flow area of the check valve. In the present calculations, $K$, $F_{m u l t}$, and $R$ are set as $2.8,1$, and 0.01 , respectively. The opening pressure difference $\Delta p_{c}$ and the maximal inner diameter of the check valve are set at $0.01 \mathrm{MPa}$ and $6 \mathrm{~mm}$, respectively. To simplify the analysis, it is assumed that the pressure waveforms are sinusoidal and the response time of check valve is short enough to be ignored. The pressure waveform of the HMPE leads that of the LMPE by a phase $\theta$. The time step is set at $1 \times 10^{-6} \mathrm{~s}$.

The direct driving sources of the gas compression and pumping in thermoacoustic compression systems are the pressure oscillations in thermoacoustic engines excited by the input thermal energy. With the thermoacoustic energy conversion effect, the input thermal energy is converted into acoustic power, which is then consumed mainly in the following ways: gas pumping through check valves, viscous and thermal-relaxation effects in thermoacoustic engines. Thus, the efficiency of the whole system is the product of thermal-to-acoustic efficiencies of thermoacoustic engines and the compression efficiency of the compression process. The thermal-to-acoustic efficiencies are strongly influenced by the design and the operation of the thermoacoustic engines. Typically, the thermal-toacoustic efficiency of a well-designed standing-wave thermoacoustic engine can reach about $0.15 \sim 0.20$ [24]. As this study mainly focuses on the characteristics of gas compression processes, the thermal-to-acoustic efficiencies of the thermoacoustic engines are not discussed thoroughly here. For simplicity, the compression efficiency $\eta$ of the OTCS is defined as the theoretical minimum work requirement $W_{i}$ divided by the actual acoustic power consumption $W_{d}$, which is actually an indicator of the perfection degree of the gas compression process,

$$
\eta=\frac{W_{i}}{W_{d}}
$$

The theoretical minimum work required is given by the isothermal compression process,

$$
W_{i}=\dot{m} R_{g} T_{0} \ln \frac{p_{H, m}}{p_{L, m}} .
$$

Neglecting the acoustic power dissipation in the connecting tubes, the actual acoustic power consumption $W_{d}$ in a thermoacoustic compression system can be expressed as

$$
W_{d}=E_{c v}+E_{L}+E_{H}
$$


where $E_{c v}, E_{L}$ and $E_{H}$ are the acoustic power dissipation terms in check valve, LMPE and HMPE. The acoustic power dissipation in check valve $E_{c v}$ can be calculated by

$$
E_{c v}=\overline{\Delta p \cdot \dot{U}}=\frac{1}{\tau} \int_{0}^{\tau} \frac{2 R_{g} T_{0} \dot{m} \Delta p}{\left(p_{H}+p_{L}\right)} d t .
$$

For standing-wave thermoacoustic engines, the acoustic power dissipations in thermoacoustic engines can be estimated by [25]

$$
E=\int\left[\frac{1}{2} \frac{(\gamma-1) \omega A_{g} \operatorname{Im}\left[-f_{\kappa}\right]}{\gamma p_{0}}\left|p_{1}\right|^{2}+\frac{1}{2} \frac{\omega \rho \operatorname{Im}\left[-f_{v}\right]}{A_{g}\left|1-f_{v}\right|^{2}}\left|U_{1}\right|^{2}\right] d x,
$$

in which $p_{1}$ and $U_{1}$ denote local pressure and volume flow amplitudes along the axial direction. $f_{\kappa}$ and $f_{v}$ are thermal and viscous functions [25]. $A_{g}$ is the cross-sectional area of the gas flow channel. $\omega$ is the angular frequency of the engine. The distributions of the pressure and volume flow amplitudes in a standing-wave thermoacoustic engine can be calculated via thermoacoustic transfer matrix method [26-27]. When the dimensions of thermoacoustic engines and the working conditions of a specific OTCS are given, the compression efficiency $\eta$ can be calculated.

\subsection{Closed thermoacoustic compression system (CTCS)}

\subsubsection{Effect of frequency}

The effect of the operating frequency on the pressure lift $\Delta p_{m}$ of the CTCS is shown in Fig. 2. $f_{L}$ and $f_{H}$ are the operating frequencies of the LMPE and the HMPE, respectively. The pressure amplitudes of the LMPE and the HMPE are both $0.1 \mathrm{MPa}$, and the phase difference $\theta$ between the pressure oscillations is set at zero. As shown, when the frequencies are not of integer multiple relationships, the pressure lift reaches up to $0.18 \mathrm{MPa}$ and is roughly independent of the working frequencies. The pressure lifts are nearly zero when the frequencies of the LMPE and the HMPE are approximately the same. When the frequencies are approximately of other integer multiple relationships, the pressure lifts are relatively small. The areas of the relatively small pressure lifts around the lines of the integer multiple relationships decrease with the increase of the multiples, which shows that the negative effect of interger multiple relationship of frequency on the pressure lift weakens when the frequency difference gets larger. Besides, the negative effects also occur when the frequencies are of $5 / 4,4 / 3,3 / 2,5 / 3$, and $5 / 2$ relationships. This is because the pressure waveforms of the LMPE and the HMPE have less superposition area, and the effective pressure difference and opening time of check valve are very limited with these frequency relationships when the phase difference is zero. Thus, the working frequencies of the engines should avoid the above relationships in practice.

\subsubsection{Effect of pressure amplitude}

Fig. 3 shows the effect of the pressure amplitudes on the pressure lift $\Delta p_{m}$ for a CTCS. The phase difference $\theta$ is set as zero, and the frequencies of the LMPE and the HMPE are $60 \mathrm{~Hz}$ and $40 \mathrm{~Hz}$, 


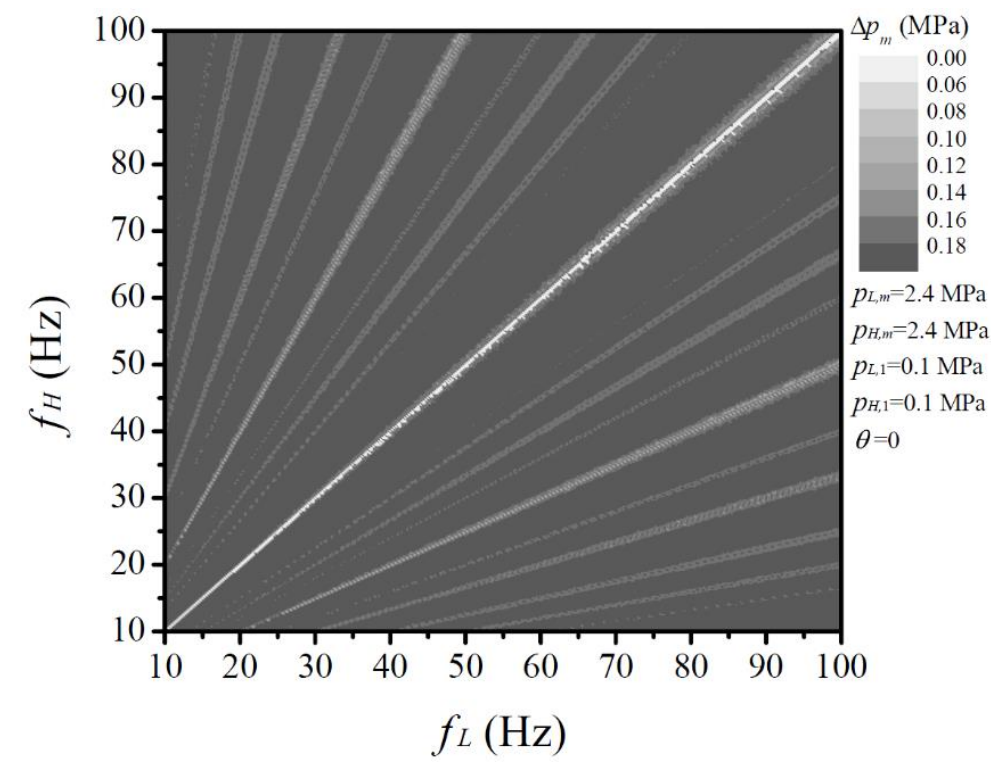

Fig. 2. Effect of frequency on pressure lift of a CTCS.

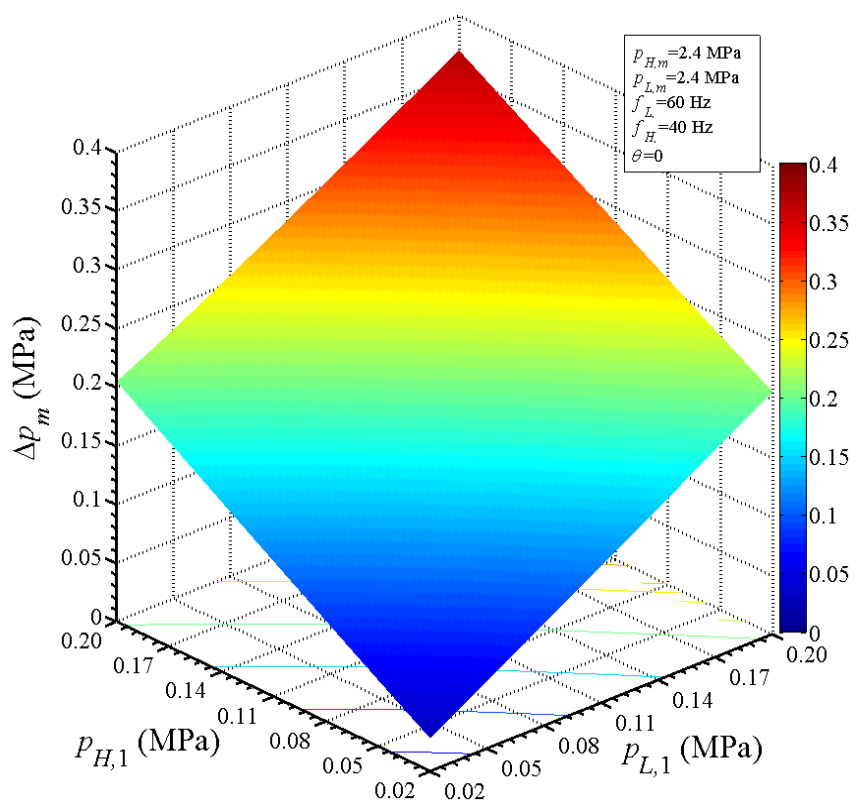

Fig. 3. Effect of pressure amplitudes on pressure lift for a CTCS.

respectively. As shown, the pressure lift is proportional to the pressure amplitudes. When the pressure amplitudes are both increased from $0.10 \mathrm{MPa}$ to $0.20 \mathrm{MPa}$, the pressure lift rises from $0.18 \mathrm{MPa}$ to $0.37 \mathrm{MPa}$. Moreover, the surface map of the pressure lift is distributed symmetrically about the plane of $p_{L, 1}=p_{H, 1}$, which indicates that the pressure amplitudes of the LMPE and the HMPE have the same effect on the pressure lift. Obviously, more gas is pumped from the LMPE to the HMPE when pressure amplitude is higher with the present calculation assumptions, which is not directly shown in Fig. 3. Thus, the pressure amplitudes should be as large as possible in practical applications and methods for increasing pressure amplitudes such as adopting pressure amplifier [12, 28-29].

Fig. 4 shows the pumping time needed to get the gas pumped from the LMPE to the HMPE at 
different pressure amplitudes. It is shown that the pumping time needed is all within several seconds, and the maximum average pumping rate of the thermoacoustic compression system is up to $5.4 \mathrm{Nm}^{3} / \mathrm{h}$.

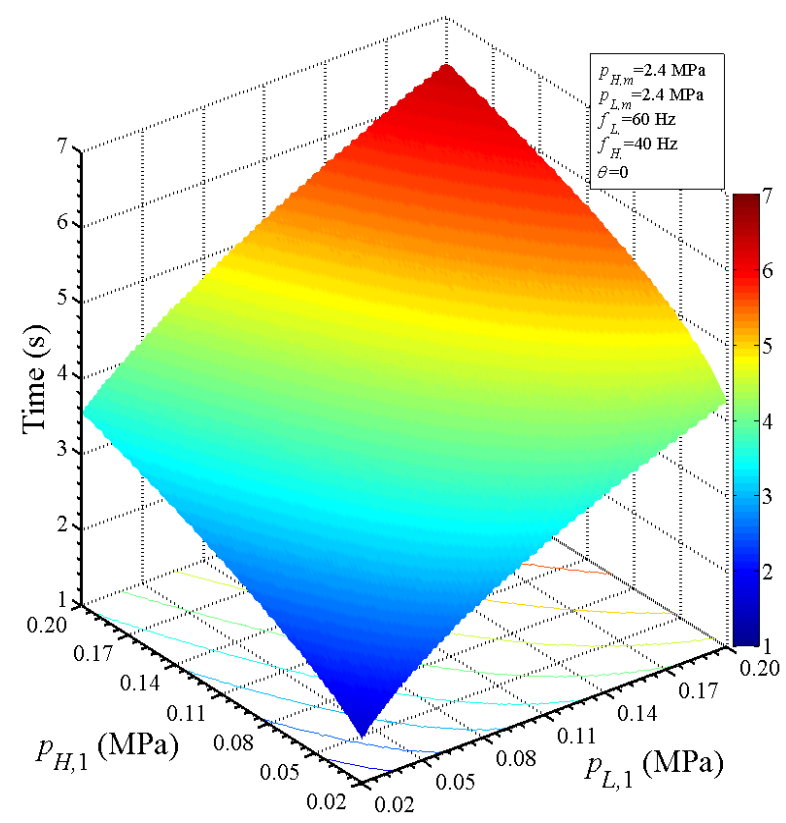

Fig. 4. Effect of pressure amplitudes on pumping time for a CTCS.

\subsubsection{Effect of phase difference}

Fig. 5 shows the effect of the phase difference $\theta$ between the pressure waveforms on the pressure lift for the CTCS. The pressure amplitudes $p_{1}$ of the LMPE and the HMPE are assumed to be the same, and the frequencies are set at $60 \mathrm{~Hz}$ and $40 \mathrm{~Hz}$, respectively. As shown, the pressure lift reaches the maximum when the phase difference $\theta$ is $\pi / 2,7 \pi / 6$ or $11 \pi / 6$, while the minimum when $\theta$ is $\pi / 6$, $5 \pi / 6$ or $3 \pi / 2$. For example, when the pressure amplitudes are both $0.156 \mathrm{MPa}$, the maximal pressure lift is 1.23 times the minimal one. A correlation for the pressure lift could be deduced from Fig. 5 as

$$
\Delta p_{m}=(|0.3560 \cdot \sin (1.5 \pi \cdot \theta-0.25 \pi)|+1.6455) \cdot p_{1}-0.01 \text {. }
$$

The above correlation indicates that the relationship between the pressure lift and the phase difference is a full-wave rectification waveform with a period of $2 \pi / 3$. This is because the transient pressure difference $\Delta p$ is a function of phase difference with a period of $2 \pi / 3$. The pressure lift reaches the maximum when the peak value of the transient pressure difference $\Delta p$ is the largest with $\theta=\pi / 2+2 k \pi / 3$, while the minimum when that of $\Delta p$ is the smallest with $\theta=\pi / 6+2 k \pi / 3$, where $k$ is an arbitrary integer. The phase relationship between the pressure waveforms of the LMPE and the HMPE are usually random, so methods to adjust the phase relationship between separately running thermoacoustic engines should be studied further to realize an efficient phase coupling [30]. Besides, the pressure lift is linearly proportional to the pressure amplitude for a given phase difference according to the correlation, which agrees with the analysis of Fig. 3. Furthermore, the pressure lift is found to have a pressure loss of $0.01 \mathrm{MPa}$ in the correlation, which is exactly the opening pressure difference $\Delta p_{c}$ of check valve. Apparently, the opening pressure difference of check valve should be decreased to 
increase the pressure lift.

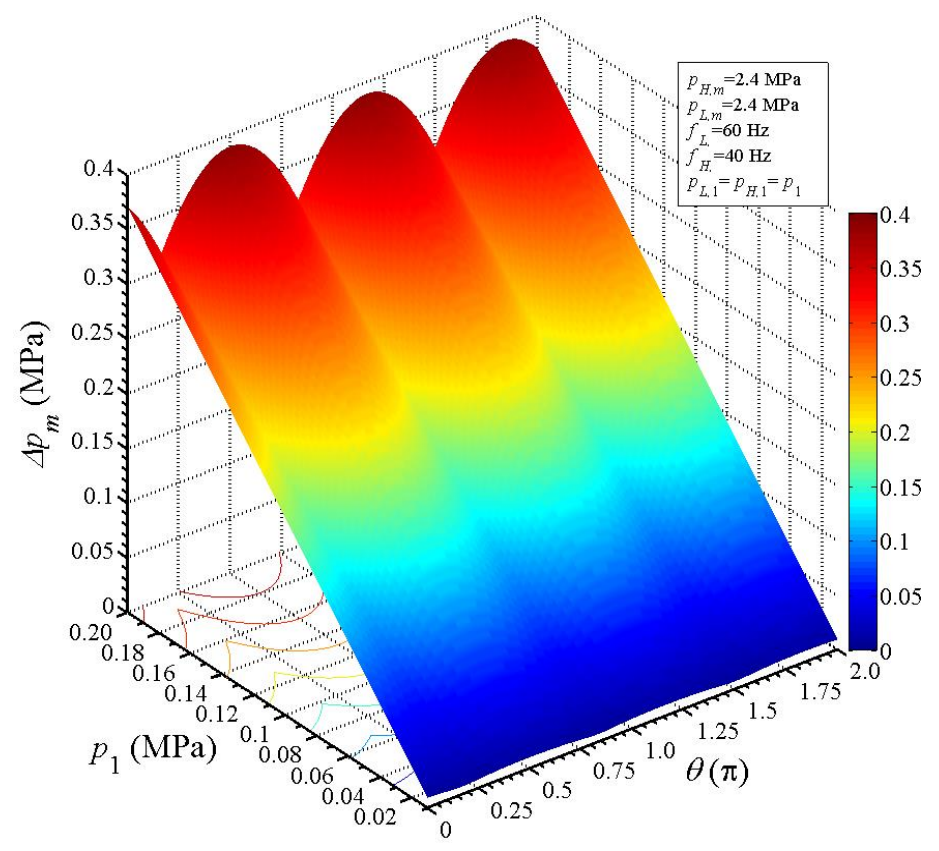

Fig. 5. Effect of phase difference on pressure lift for a CTCS.

\subsubsection{Transient process simulation of CTCS}

Based on the computation model given before, the dynamic thermoacoustic compression process of the CTCS is simulated. For the convenience of experimental validation, the geometric and operating parameters are set the same as those in the experiments, which will be presented systematically later. The experimental CTCS is composed of two standing-wave thermoacoustic engines and two reservoirs, as shown in Fig. 6. The dimensions of the engines are listed in Table 1. The volumes of LMPR and HMPR are both $10 \mathrm{~L}$. The calculated working frequencies of the two engines are around $65 \mathrm{~Hz}$ and $40 \mathrm{~Hz}$ when nitrogen is used as the working gas.

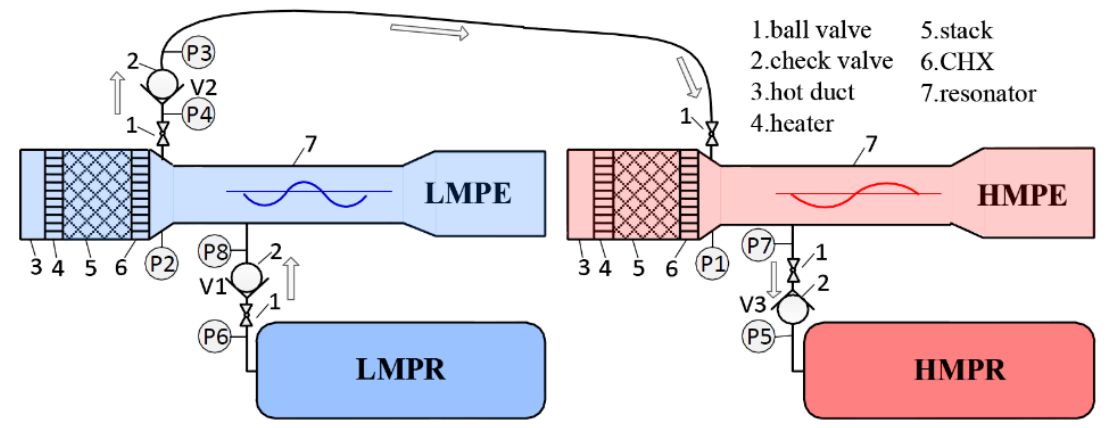

Fig. 6. Schematic of experimental setup. P1 to P8 denote pressure sensor locations. T1 and T2 denote temperature sensor locations. Gas flows in the direction as shown by arrows.

Fig. 7 shows the calculated variation trends of mean pressures and instantaneous pumping rates during the compression process at different initial mean pressures. As shown, the pressures in different parts of the system depart from each other rapidly. It takes about $30 \mathrm{~s}$ to complete the compression process in the calculation, and less than $8 \mathrm{~s}$ to complete $90 \%$ of the final pressure lift. 
Table 1. Geometric parameters of the LMPE and HMPE. ( $D \times L$ : diameter $\times$ Length; $\varphi$ : porosity; $r_{h}$ : hydraulic radius). The units for length, diameter and radius are mm.

\begin{tabular}{lcccccc}
\hline \multirow{2}{*}{ Components } & \multicolumn{3}{c}{ LMPE } & \multicolumn{3}{c}{ HMPE } \\
\cline { 2 - 7 } & $D \times L$ & $\varphi$ & $r_{h}$ & $D \times L$ & $\varphi$ & $r_{h}$ \\
\hline Hot duct & $50 \times 70$ & 1 & $D / 4$ & $59.5 \times 60$ & 1 & $D / 4$ \\
Heater & $50 \times 80$ & 0.3 & $2 \times 10^{-3}$ & $59.5 \times 50$ & 0.35 & $4 \times 10^{-3}$ \\
Stack & $50 \times 60$ & 0.84 & $3.6 \times 10^{-4}$ & $59.5 \times 110$ & 0.4 & $3 \times 10^{-4}$ \\
CHX & $50 \times 60$ & 0.18 & $9.5 \times 10^{-4}$ & $59.5 \times 40$ & 0.25 & $1.5 \times 10^{-3}$ \\
Resonator & $32 \times 1050$ & 1 & $D / 4$ & $39 \times 2600$ & 1 & $D / 4$ \\
Compliance cavity & $81 \times 850$ & 1 & $D / 4$ & $85 \times 500$ & 1 & $D / 4$ \\
\hline
\end{tabular}

(a)

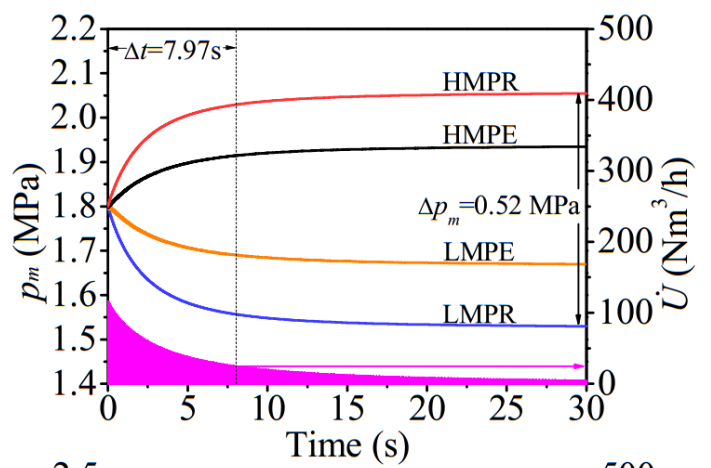

(b)
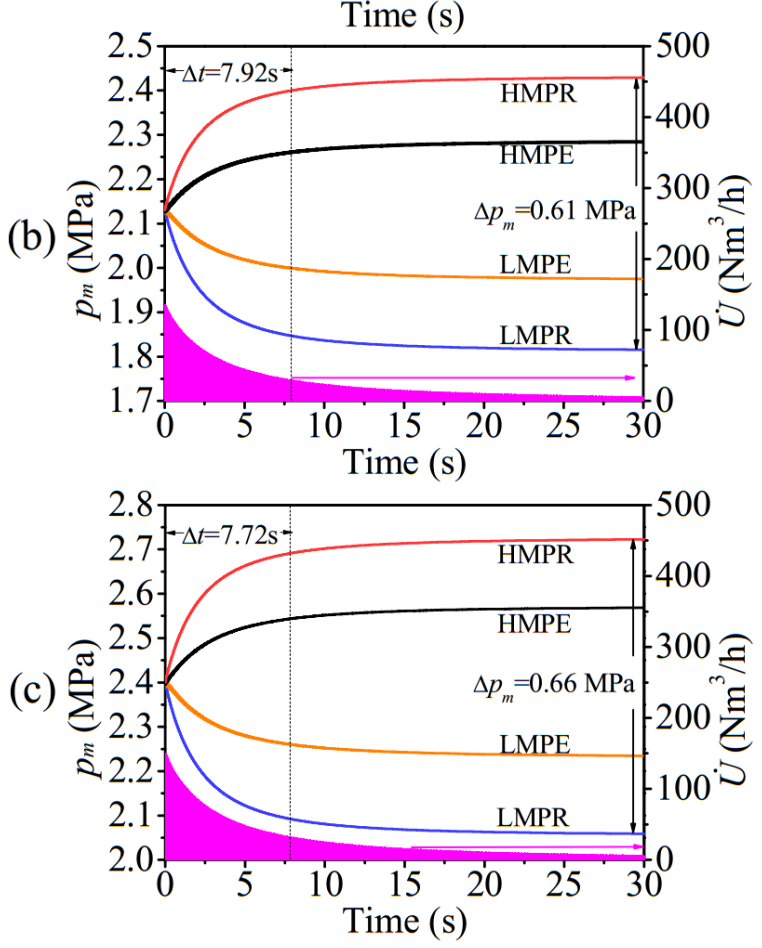

Fig. 7. Variations of mean pressures $p_{m}$ and instantaneous pumping rates $\dot{U}$ during compression at initial mean pressures of (a) $1.80 \mathrm{MPa}$, (b) 2.13 MPa, and (c) $2.40 \mathrm{MPa}$ in calculations. The pressure amplitudes used in calculations are set the same as in the experiments before compression process starts. 
The highest instantaneous pumping rate from the LMPR to the LMPE can reach up to $115 \mathrm{Nm}^{3} / \mathrm{h}$ at 1.80 $\mathrm{MPa}$ and $150 \mathrm{Nm}^{3} / \mathrm{h}$ at $2.4 \mathrm{MPa}$. The calculated mean pressure lift $\Delta p_{m}$ increases with the initial mean pressure, and achieves $0.66 \mathrm{MPa}$ when the initial mean pressure is $2.40 \mathrm{MPa}$.

\subsection{Open thermoacoustic compression system (OTCS)}

For a CTCS, gas is always restricted inside it, and gas compression stops once the pressure difference becomes not high enough to open the check valve. Different from the operation of CTCS, OTCS continuously pumps gas from a low pressure gas source to a high pressure one. The mean pressure in every stage of an OTCS stays constant when it is operated steadily, which maintains an effective pressure difference to make the compression system work continuously. Continuous gas pumping and even novel energy conversion systems may be developed based on the principle of OTCS. For a steadily operated OTCS, pumping rate is of great importance to evaluate the performance, which is quite different from the CTCS. Effects of frequency, pressure amplitude, phase difference, and mean pressure difference on the average pumping rate are calculated and analyzed.

\subsubsection{Effect of frequency}

Fig. 8 shows the effect of the operating frequency on the average pumping rate $U$ for an OTCS. The mean pressures $p_{L, m}$ and $p_{H, m}$ of the LMPE and the HMPE are 2.3 MPa and 2.4 MPa, respectively. The pressure amplitudes are both $0.2 \mathrm{MPa}$ and the phase difference $\theta$ between the pressure waveforms is zero. As shown, the pumping rate $U$ is around $50.0 \mathrm{Nm}^{3} / \mathrm{h}$ at most frequencies, which indicates a weak effect of the frequencies on the pumping rate. However, when the frequencies are the same, the pumping rate drops down to zero, which indicates that no effective superposition area is generated by the pressure waveforms at this operating point. The pumping rate is low when the frequencies are of 2 and 3 times relationships. For example, the pumping rate is about $47.9 \mathrm{Nm}^{3} / \mathrm{h}$ when the relationship is 2 times, and it is only $40 \mathrm{Nm}^{3} / \mathrm{h}$ when the relationship is 3 times. This is due to the reduced superposition areas of the pressure waveforms of the LMPE and HMPE. Different from CTCS, when the operating frequency is of larger integer multiple relationships than 3 , this influence becomes very

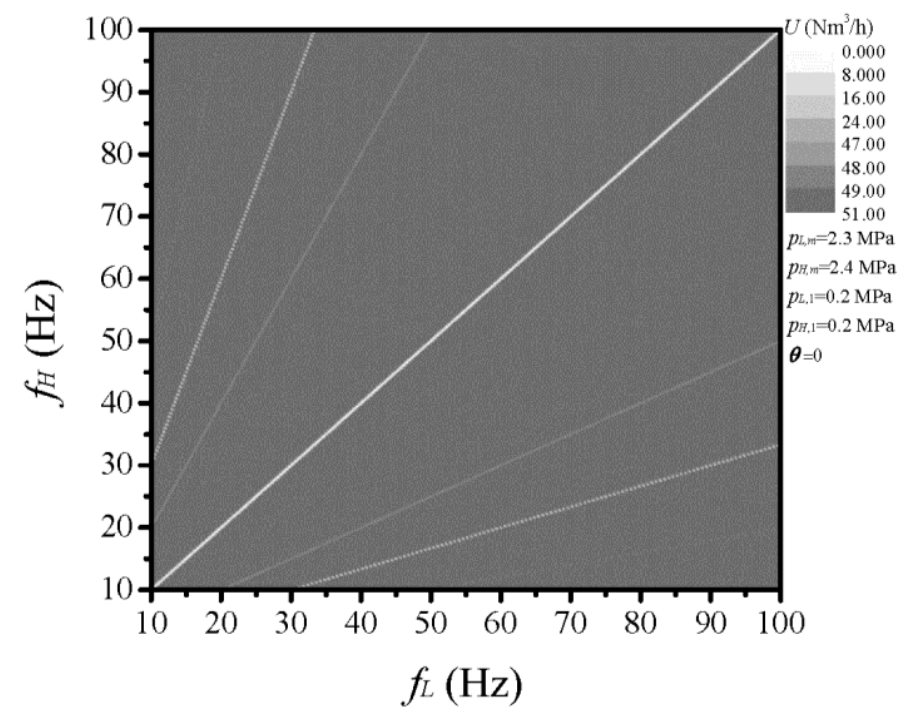

Fig. 8. Effect of operating frequency on pumping rate for an OTCS. 
weak and even vanishes because the superposition area resumes. So, the selection of operating frequencies of the thermoacoustic engines should avoid the above three integer multiple relationships when designing an OTCS.

\subsubsection{Effect of pressure amplitude}

Fig. 9 shows the effect of pressure amplitude on the average pumping rate $U$. The pressure amplitudes of the LMPE and the HMPE are assumed to be the same for simplicity. It is clearly shown that the pumping rate $U$ rises nearly linearly with the pressure amplitude. When the pressure amplitude is increased from $0.1 \mathrm{MPa}$ to $0.2 \mathrm{MPa}$, the pumping rate rises from $16.15 \mathrm{Nm}^{3} / \mathrm{h}$ to 49.74 $\mathrm{Nm}^{3} / \mathrm{h}$, corresponding to an increase of $208.0 \%$. Therefore, the output capability of thermoacoustic engines is critical to the performance of the OTCS. Generally speaking, the pressure amplitude of an efficient travelling wave thermoacoustic engine is frequently above $0.3 \mathrm{MPa}$ [15, 31-32], which is big enough to generate a pumping rate of $72.5 \mathrm{Nm}^{3} / \mathrm{h}$.

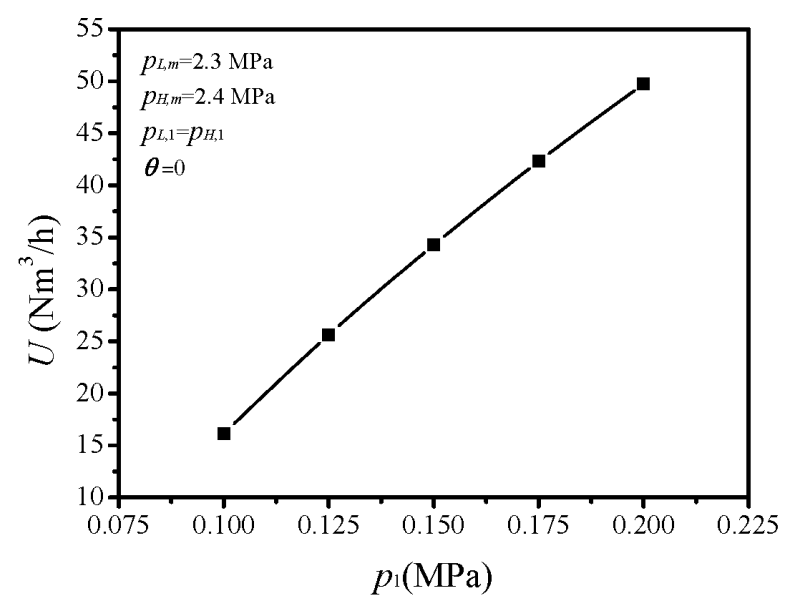

Fig. 9. Effect of pressure amplitude on average pumping rate for an OTCS.

\subsubsection{Effect of phase difference}

The effect of the phase difference between pressure waveforms on the average pumping rate is shown in Fig. 10. The frequencies of the LMPE and the HMPE are $60 \mathrm{~Hz}$ and $40 \mathrm{~Hz}$, respectively. As shown, the variation trend of the pumping rate repeats with a period of $2 \pi / 3$. When the pressure amplitude is smaller than about $0.09 \mathrm{MPa}$, the pumping rate reaches the maximum at a phase difference of $\pi / 2,7 \pi / 6$, and $11 \pi / 6$, while the minimum when it is $\pi / 6,5 \pi / 6$, and $3 \pi / 2$. In contrast, the variation trend changes completely when the pressure amplitude exceeds $0.09 \mathrm{MPa}$. Calculation of the superposition area at different pressure amplitudes, as illustrated by the shadows in Fig. 1, shows that it has the above mentioned variation tendency, and further determines the distribution of pumping rate in Fig. 10. 


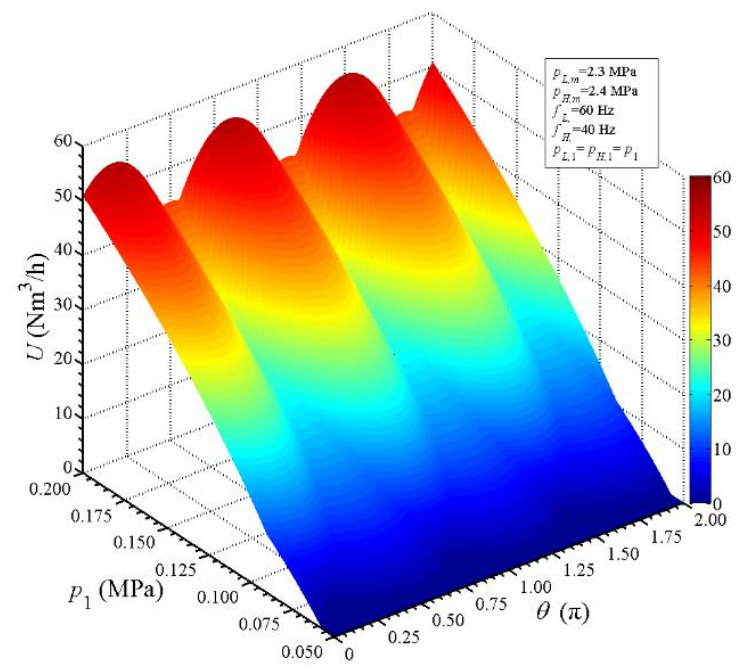

Fig. 10. Effect of phase difference on pumping rate for an OTCS.

\subsubsection{Effect of mean pressure difference}

Fig. 11 shows the effect of the mean pressure difference $\Delta p_{m}=p_{H, m}-p_{L, m}$ on the pumping rate of the OTCS, which can be considered as the performance curve like that of traditional pumps or fans. The mean pressure of the high-pressure side $p_{H, m}$ is assumed to be fixed at $2.4 \mathrm{MPa}$. As shown, for given pressure amplitudes, the pumping rates increases as the mean pressure difference $\Delta p_{m}$ decreases. Gas pumping can be easily realized at very low pressure amplitude when the mean pressure difference $\Delta p_{m}$ is relatively small. For example, when the mean pressure difference $\Delta p_{m}$ is $0.05 \mathrm{MPa}$ and the pressure amplitude $p_{1}$ is $0.2 \mathrm{MPa}$, the pumping rate reaches up to $65.37 \mathrm{Nm}^{3} / \mathrm{h}$. As the mean pressure difference $\Delta p_{m}$ increases, the pumping rate decreases, and larger pressure amplitudes are needed to get the gas pumped. Therefore, in order to achieve a certain pressure lift with a relatively high pumping rate, a trade-off should be made between them.

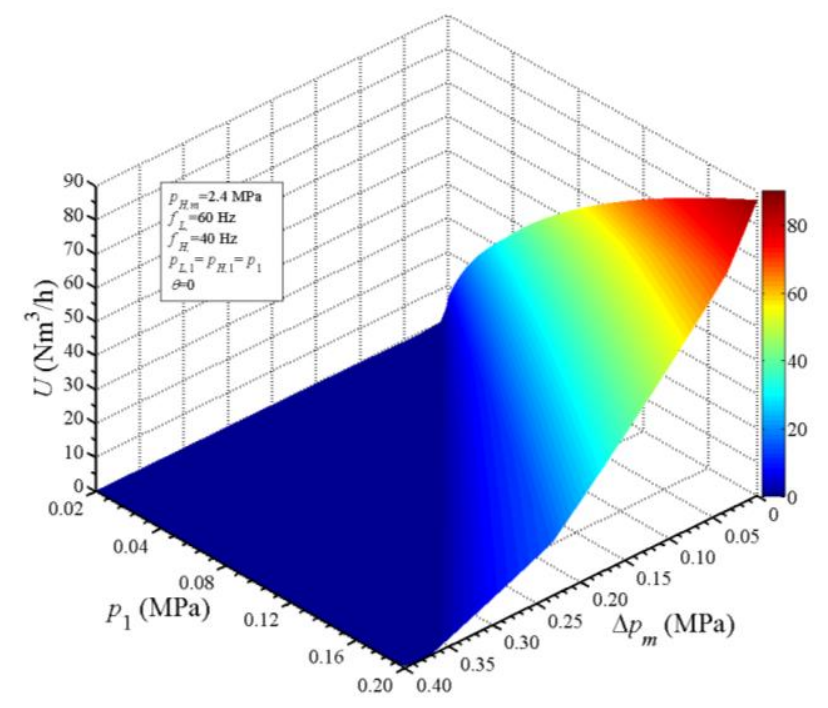

Fig. 11. Effect of mean pressure difference on pumping rate for an OTCS. 


\subsubsection{Efficiency Analysis of OTCS}

For efficiency analysis, take a specific OTCS as an example, which is composed of the two standing-wave thermoacoustic engines used in the following experimental study. Fig. 12 shows the effect of pressure amplitude on compression efficiency $\eta$. The mean pressures of the LMPE and HMPE are set at 2.3 MPa and 2.4 MPa, respectively. The pressure amplitudes of the LMPE and HMPE are the same. The phase difference between the engines are set as zero. As shown, the compression efficiency reaches the maximum value of about 0.57 when the pressure amplitude is $0.092 \mathrm{MPa}$. When the pressure amplitude increases, the difference between the acoustic power consumption of the OTCS and the ideal compression work becomes larger, and the compression efficiency thus decreases. For example, when pressure amplitude is $0.20 \mathrm{MPa}$, the ideal compression work is $46.3 \mathrm{~W}$ while that actually required by the OTCS is $149.3 \mathrm{~W}$, and the compression efficiency is 0.31 . It should be noted that the work required by the OTCS is strongly related to the thermoacoustic engines selection. If more compact thermoacoustic engines utilizing mechanical resonators are used instead of the present engines, the actually required acoustic work would be remarkably reduced as less acoustic power dissipations occur inside the engines.

Fig. 13 shows the effect of the mean pressure difference between the LMPE and the HMPE on the compression efficiency. When the pressure amplitude is fixed, the compression efficiency first increases and then decreases with the mean pressure difference $\Delta p_{m}$. When the mean pressure difference is zero, which means the gas is not "compressed" from a low mean pressure to a high one, the compression efficiency is zero according to its definition. When the mean pressure difference is so large that it exceeds the sum of the pressure amplitudes of the LMPE and HMPE, for instance, $0.25 \mathrm{MPa}$ when pressure amplitude is $0.1 \mathrm{MPa}$, the efficiency is also zero because the pressure of the LMPE can never get higher than that of the HMPE and the gas cannot be pumped from the LMPE to the HMPE. When the mean pressure difference is fixed, there also exists optimal pressure amplitude for the compression efficiency, which coincides with the conclusions of Fig. 12. As shown by the dark red region in Fig. 13, it is beneficial for the compression efficiency when the mean pressure difference and the pressure amplitude are both small. The highest compression efficiency reaches about 0.63 .

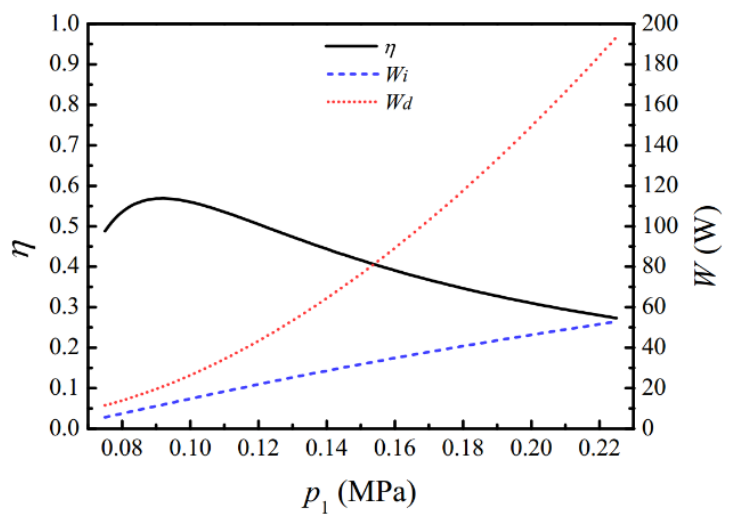

Fig. 12. Effect of pressure amplitude on compression efficiency $\eta$. 


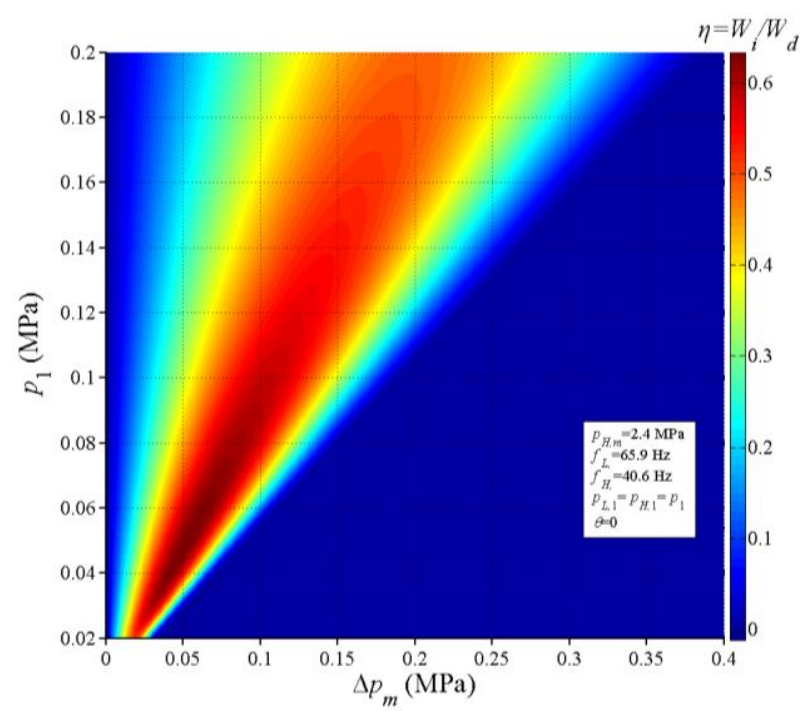

Fig. 13. Effect of mean pressure difference on compression efficiency $\eta$.

\section{Experimental results}

As shown in Fig. 6, a demonstrative CTCS composed of two standing-wave thermoacoustic engines and two reservoirs was constructed and tested. The two thermoacoustic engines whose working frequencies are about $64 \mathrm{~Hz}$ and $40 \mathrm{~Hz}$ with nitrogen as the working gas are chosen as the LMPE and the HMPE, respectively. The distribution of pressure lift in Fig. 2 shows that the pressure lift at these two frequencies is not negatively affected. Check valves V1 and V3 with an opening pressure of 0.01 MPa were supplied by HEROSE (type: 05011.X.0300), and check valve V2 with an opening pressure of 0.003-0.021 MPa was supplied by Swagelok (type: SS-6C-MM-1/3). Eight calibrated piezoresistive pressure sensors with an accuracy of $\pm 0.3 \%$ and two calibrated NiCr-NiSi thermocouples were installed to measure pressures and heating temperatures, respectively. The ball valves were opened one by one when the thermoacoustic engines worked steadily. Because of the time required to open the ball valves, time lags between them occured during the experiments.

According to the theoretical analysis, larger pressure amplitudes at the two ends of check valves are beneficial to thermoacoustic compression and pumping effect. In the experiments, three measures are taken to obtain as high pressure amplitude as possible. Firstly, the LMPE and the HMPE both work at the maximum allowable heating temperature of approximately $650{ }^{\circ} \mathrm{C}$ to achieve a large temperature ratio between the stack ends. Secondly, connecting points for the connecting tubes are set at positions where the pressure amplitudes are relatively large. As a result, the connecting points for the transfer tube between the engines are set near the CHXs, while the ones for the tubes connecting the reservoirs are set between the midpoints of the resonators and the CHXs. Thirdly, pressure amplifier is adopted to amplify the pressure amplitude. The length of the tube connecting the HMPE and the check valve V2 is set as $3 \mathrm{~m}$ by experimental optimization, which is an appropriate length to amplify the pressure amplitude in the HMPE without degrading the performance of the thermoacoustic engine much.

Figs. 14(a)-(c) show the variations of mean pressures $p_{m}$ and pumping rates $U$ from the LMPR 
to the LMPE during the thermoacoustic compression at different initial mean pressures. The pumping rate $U$ is calculated based on the mean pressure variation in the LMPR according to the ideal gas equation of state. As shown, the mean pressures in the four components depart from each other immediately after the compressions start, whose variation trends agree well with the transient process simulation in Fig. 7. As stated above, the check valves don't start to work simultaneously in experimental operations, but one by one from V2, V3 to V1 within several seconds. As shown in Fig. 14(a), when the check valve V2 is first opened, the mean pressure in the LMPE goes down sharply while that in the HMPE rises subsequently. Four seconds later when the check valve V3 is opened, the gas in the HMPE is pumped into the HMPR, resulting in a little decrease of the mean pressure in

(a)

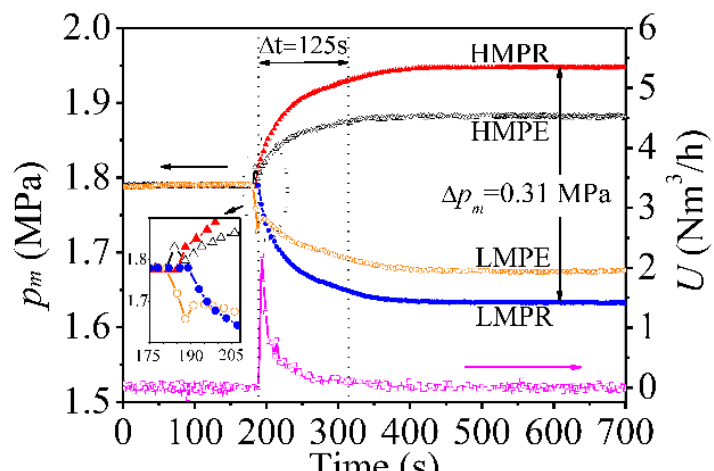

(b)

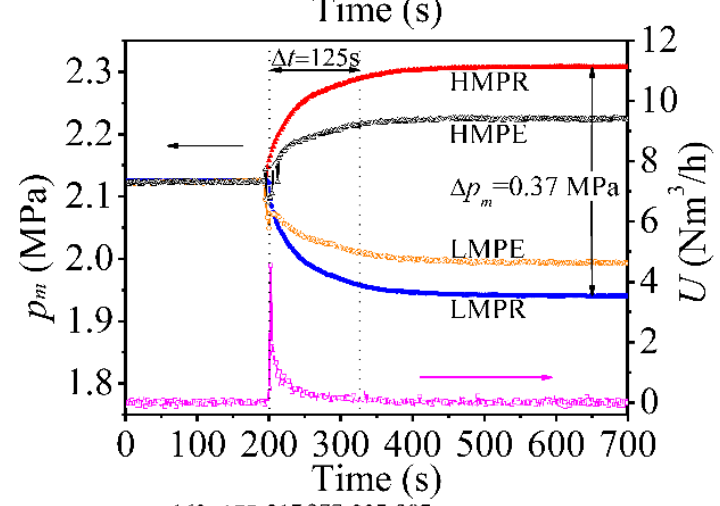

(c)

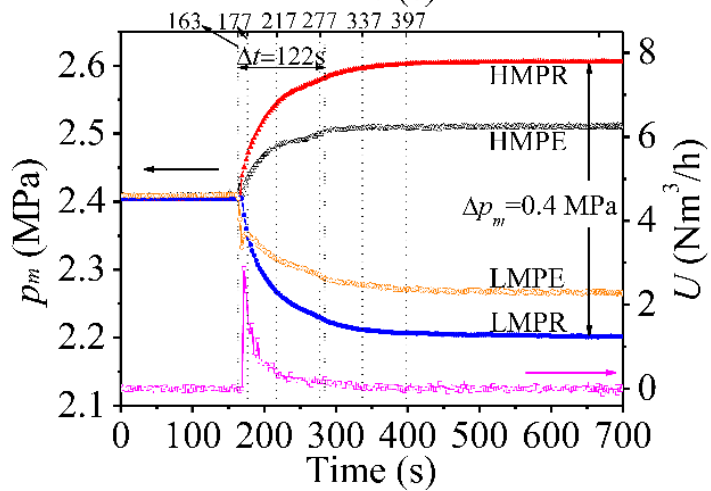

Fig. 14. Variations of mean pressures $p_{m}$ and pumping rates $U$ during compression at initial mean pressures of (a) 1.80 $\mathrm{MPa}$, (b) $2.13 \mathrm{MPa}$, and (c) $2.40 \mathrm{MPa}$ in experiments. When the initial mean pressure is $2.13 \mathrm{MPa}$, the opening of check valve V2 is 7 seconds ealier that that of check valve V1, which results in the decrease of the mean pressure of LMPE. Thus, larger mean pressure difference between LMPR and LMPE occurs before the gas pumping out of LMPR starts. When the initial mean pressure is $2.40 \mathrm{MPa}$, the time gap between the opening of the two valves is only 6 seconds. Gas pumped out of LMPE is not as much as that in $2.13 \mathrm{MPa}$. As a result, the mean pressure difference between LMPR and LMPE is smaller, and the maximum pumping rate in Fig.14(c) is a little lower than that in Fig.14(b). 
the HMPE and continuous increase of the mean pressure in the HMPR. Check valve V1 is opened another four seconds later, and the gas in the LMPR starts to be pumped into the LMPE. Thus, the mean pressure in the LMPE increases a little after that. The change rates of the mean pressures and the pumping rate $U$ slow down as the pressure differences increase since the superposition areas of the pressure waveforms decrease. The variation trends of the mean pressures shown in the other two figures are very similar. The average pumping rate $U$ reaches up to $4.55 \mathrm{Nm}^{3} / \mathrm{h}$ during the first two seconds when the initial mean pressure is $2.13 \mathrm{MPa}$, as shown in Fig. 14(b). As the time gap between the opening of valve V2 and V1 for $2.13 \mathrm{MPa}$ is one second longer than that for $2.4 \mathrm{MPa}$, more gas is pumped out of LMPE for $2.13 \mathrm{MPa}$, which results in a larger mean pressure difference between LMPR and LMPE before the gas pumping out of LMPR starts. Therefore, the maximum pumping rate for $2.4 \mathrm{MPa}$ is a little lower than that for $2.13 \mathrm{MPa}$. The pressure lift reaches $0.4 \mathrm{MPa}$ when the initial mean pressures are $2.40 \mathrm{MPa}$, as shown in Fig. 14(c). For the three cases, the time needed to complete $90 \%$ of the final pressure lift between the LMPR and the HMPR is less than $125 \mathrm{~s}$.

The measured and calculated final mean pressure difference generated between the LMPR and the HMPR, and the average pumping rates during the $90 \%$ of the compression processes of the above three cases are given in Fig. 15 with respect to the initial mean pressure. As shown, the measured pressure amplitudes $p_{H, 1}$ and $p_{L, 1}$ of the HMPE and the LMPE increases with the mean pressure. As a result, the final mean pressure lift and average pumping rate in the calculation and the experiments both increase with the mean pressure. The variation trends of the calculated pressure lift and average pumping rate agree well with the experimental ones. When the mean pressure is $2.40 \mathrm{MPa}$, the pressure lifts in the calculation and the experiment are $0.66 \mathrm{MPa}$ and $0.40 \mathrm{MPa}$, respectively. The deviations of the average pumping rate are much more noticeable. The calculated average pumping rate is $14.12 \mathrm{Nm}^{3} / \mathrm{h}$ while the measured one is only $0.51 \mathrm{Nm}^{3} / \mathrm{h}$. The deviations is mainly due to the pressure waveform deformations occurred in experiments, which will be discussed later. As the pressure waveforms are so dramatically deformed from sinusoidal shapes that the effective pressure differences needed to pump gas are severely affected. Secondly, the pressure amplitudes in the engines are also decreased because of the compression process. Finally, the check valves in the real system are different from the idealized ones used in modelling.

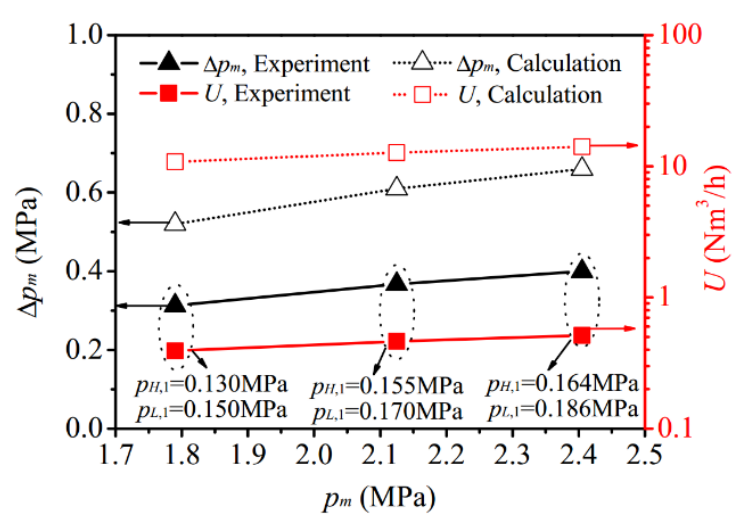

Fig. 15. Final mean pressure difference between LMPR and HMPR, and average pumping rate during $90 \%$ of compression process vs. initial mean pressures. 
The input heating powers of the LMPE and HMPE before and after the thermoacoustic compression starts in the experiments are given in Table 2. The heating powers are adjusted to keep the heating temperatures stable at around $650{ }^{\circ} \mathrm{C}$. As shown, the total heating powers are roughly 1.8 2.0 kW before the compression starts. After the compression process starts, the heating power decreases to about $1.5 \sim 1.7 \mathrm{~kW}$, due to the decreases of the pressure amplitudes inside the engines. It should be noted that the heating power input of the demonstrative system is still large at present, due to the poor heat transfer performances of the heater, the limited energy conversion efficiencies of the thermoacoustic engines. Besides, the volumes of thermoacoustic engines are relatively large, which results in a large amount of acoustic power dissipation. The efficiency of the whole system can be increased if much more compact thermoacoustic engines which can provide the same pressure amplitude are used.

Table 2. Heating powers of LMPE and HMPE before and after thermoacoustic compression starts.

\begin{tabular}{ccccc}
\hline \multirow{2}{*}{$\begin{array}{c}\text { Initial mean } \\
\text { pressure }\end{array}$} & \multicolumn{2}{c}{$\begin{array}{c}\text { Heating power before } \\
\text { compression starts, W }\end{array}$} & \multicolumn{2}{c}{$\begin{array}{c}\text { Heating power after } \\
\text { compression starts, W }\end{array}$} \\
\cline { 2 - 5 } & LMPE & HMPE & LMPE & HMPE \\
\hline $1.80 \mathrm{MPa}$ & 1016 & 845 & 909.7 & 600.2 \\
$2.13 \mathrm{MPa}$ & 1024 & 901.7 & 1059 & 660 \\
$2.40 \mathrm{MPa}$ & 1092 & 912 & 1064 & 632 \\
\hline
\end{tabular}

Fig. 16 shows the pressure waveforms in the HMPE (P1) and the LMPE (P2) when the initial mean pressure is at $2.4 \mathrm{MPa}$, and the timings are denoted accordingly in Fig. 14(c). The gas pumping starts at $165 \mathrm{~s}$ when check valve V2 is first opened. The pressure waveforms are both in good sinusoidal shapes around the same mean pressure at $164 \mathrm{~s}$. Once the gas pumping starts, the waveform in the HMPE is drastically deformed by the large direct gas flow in the first several seconds, as shown at $166 \mathrm{~s}$ and $169 \mathrm{~s}$. A little deformation of the lower part of the waveform still exists $217 \mathrm{~s}$ later. When the pressure of the HMPE is higher than that of the LMPE, the check valve has no chance to be opened. Thus, the deformation of the higher part of the waveform in the HMPE is less serious compared to that of the lower part. Almost no deformation of the pressure waveform in the LMPE occurs during the whole gas pumping process. The reason may be that the check valve $\mathrm{V} 2$ is much closer to the LMPE and the tube connecting to the HMPE is much longer than that to the LMPE. As a result, the long tube connected to the HMPE acts as a pressure amplifier and has a strong influence on the performance of the HMPE.

Figs. 17 and 18 show the pressure waveforms before and after the check valves V1 and V2. As shown in Fig. 17, the pressure P6 in the LMPR oscillates at the same frequency as the LMPE after the check valve V1 is opened, and the phase difference between P8 and P6 increases with the time. The mean pressure in the LMPR changes very little from $337 \mathrm{~s}$ to $397 \mathrm{~s}$, and the pressure oscillation in the LMPR almost vanishes at $397 \mathrm{~s}$, which means the gas pumping from the LMPR to the LMPE 
nearly stops even though remarkable pressure difference still exists. A maximum instantaneous pressure difference of about $0.07 \mathrm{MPa}$ between P8 and P6 at $397 \mathrm{~s}$ indicates that the actual opening pressure difference of the check valve V1 is much larger than the nominal one, which affects the pressure lift significantly. The pressure amplitude of P8 rarely has any change during the process, indicating the steady performance of the LMPE during the gas pumping process.
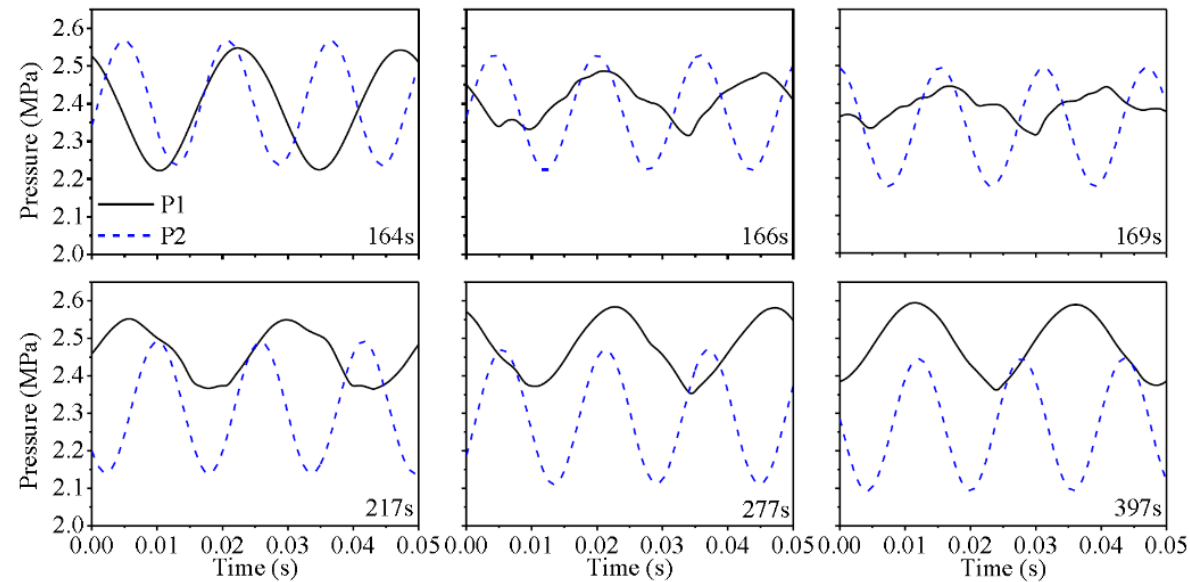

Fig. 16. Pressure waveforms in HMPE and LMPE during thermoacoustic compression process. The gas pumping starts at $165 \mathrm{~s}$ when check valve $\mathrm{V} 2$ is opened.

Fig. 18 shows the pressure waveforms before (P4) and after (P3) the check valve V2. The pressure waveform of P3 after the check valve is also seriously deformed as that in the HMPE (P1) as shown in Fig. 16. Besides, the pressure waveform of P3 is more seriously influenced at the lower part when the gas is sucked from the LMPE side into the HMPE side. Moreover, the peak-to-peak amplitude of $\mathrm{P} 3$ is about $0.31 \mathrm{MPa}$ while that of P1 is only $0.18 \mathrm{MPa}$ at $217 \mathrm{~s}$, which indicates the pressure amplification effect of the pressure amplifier. The pressure waveform of P4 is also slightly deformed, due to the much higher direct gas flow rate within the thin connecting tube than that in the LMPE. In brief, the gas pumping results in the pressure waveform deformations in the thermoacoustic compression system, which is not taken into account in previous calculation yet. The deformed

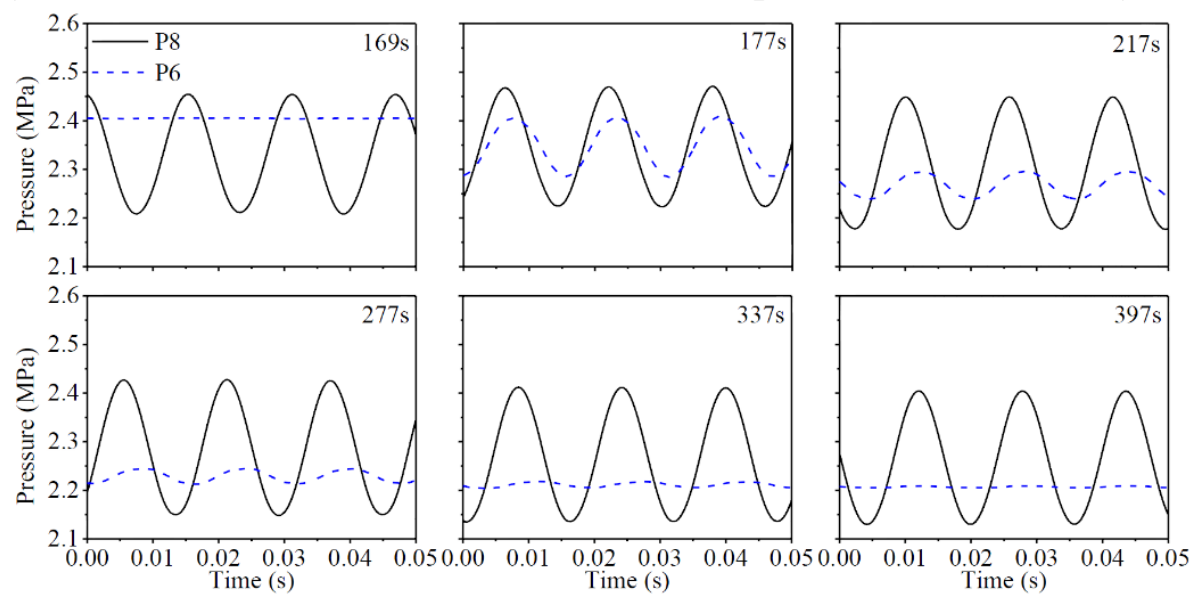

Fig. 17. Pressure waveforms before and after check valve V1 during thermoacoustic compression process. Check valve V1 opens at $172 \mathrm{~s}$. 


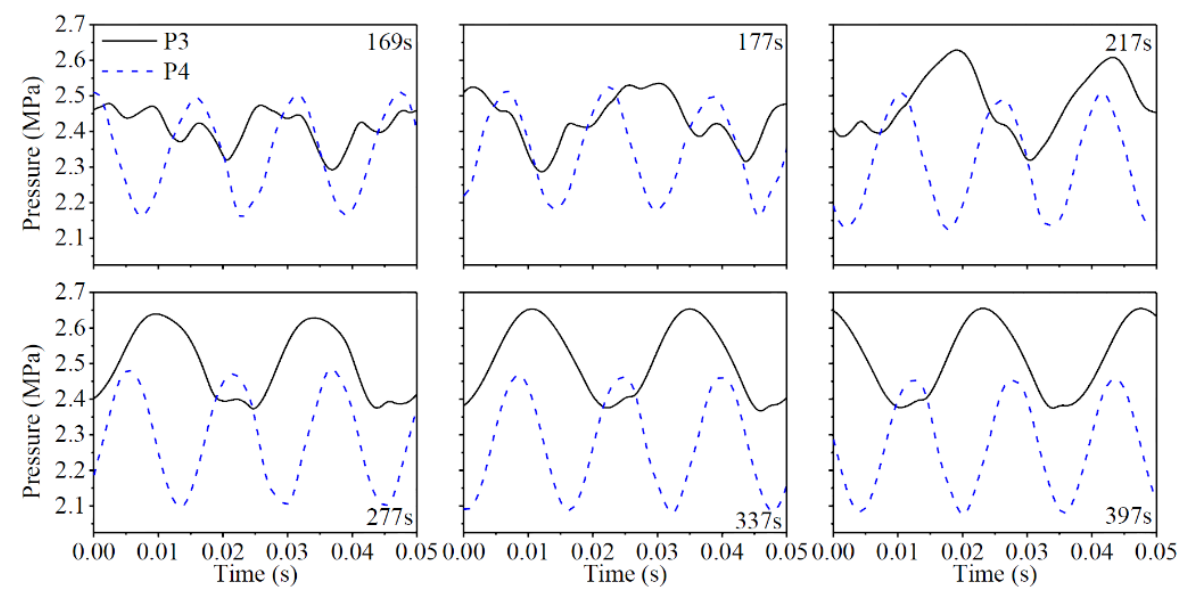

Fig. 18. Pressure waveforms before and after check valve V2 during thermoacoustic compression process. Check valve V2 opens at $165 \mathrm{~s}$.

pressure waveforms then decrease the effective pressure differences across the check valves, and affect the gas pumping rate negatively in turn. Thus, these two factors lead to the formation of a negative feedback system, and make the gas pumping rate fall in a reasonable range.

Till now, the key factors that affect the performance are revealed. It is shown that the performance can be further improved in the following aspects. Firstly, more powerful, efficient and compact thermoacoustic engines should be designed to enlarge pressure amplitudes and decrease acoustic power losses. Pressure amplifier can be further optimized to amplify pressure amplitudes without affecting the thermoacoustic conversion too much. Secondly, the flow resistance, the opening pressure difference, and the response time of the check valves should be minimized to reduce undesirable losses. Thirdly, effective approaches to weaken the influence of pressure waveform deformations before and after check valves need to be explored. Last but not least, the coupling mechanism of thermoacoustic engines and check valves should be further investigated. Efficient thermoacoustic conversion exposed both to alternating and direct gas flows should be studied.

\section{Conclusions}

We have proposed a thermoacoustic compression effect based on the pressure oscillation nature of thermoacoustic engines and the gas flow rectification effect of the check valves. On the basis of the effect, closed and open thermoacoustic compression systems can be realized to get gas compressed and pumped. Theoretical analyses and computational results have shown the application potential of such systems for its remarkable merits, such as large pumping rate, high pressure lift, simple configuration, thermally driven nature, and oil-free operation.

Computation shows that the operating frequencies of the thermoacoustic engines should not be of small integer multiple relationships for efficient operation. Large pressure amplitudes are beneficial to the performance of both the closed and the open systems. It is also shown that pressure amplitude and mean pressure difference between the low and high mean pressure engines should be optimized to increase the compression and pumping efficiency. Besides, it is beneficial for the 
compression efficiency if more compact thermoacoustic engines with high pressure amplitudes are used in an open system. A demonstrative closed thermoacoustic compression system are then experimentally studied. The variation trends of the mean pressures during the compression process, final pressure lifts, and average pumping rate in the calculations and the experiments agree well. In the experiments, a maximum average pumping rate of $4.55 \mathrm{Nm}^{3} / \mathrm{h}$ and a maximum pressure lift of 0.4 MPa were achieved when the initial mean pressures were $2.13 \mathrm{MPa}$ and 2.40 MPa, respectively. Furthermore, it is found that the direct gas flow can cause serious pressure waveform deformation, which then affects the gas pumping negatively in turn.

\section{Acknowledgements}

This research is financially supported by the Major State Basic Research Development Program of China (973 Program) under contract No. 2010CB227303 and National Natural Science Foundation of China under contract No. 61077035.

\section{References}

[1] McMahon, H.O., Gifford, W.E., A New Low-Temperature Gas Expansion Cycle -Part I, in Advances in Cryogenic Engineering1960, Plenum Press: New York. p. 354-366.

[2] Sun, D.M., Xu, Y., Chen H.J.,Shen, Q., Zhang, X.J., Qiu, L.M.,Acoustic characteristics of a mean flow acoustic engine capable of wind energy harvesting: Effect of resonator tube length. Energy, 2013. 55:p. 361-368.

[3] Sun, D.M., Xu, Y., Chen, H.J., Wu, K., Liu, K.K., Yu, Y., A mean flow acoustic engine capable of wind energy harvesting. Energy Conversion and Management, 2012. 63(0): p. 101-105.

[4] Yu, Y., Sun, D.M., Wu, K., Xu, Y., Chen, H.J., Zhang, X.J., Qiu, L.M., CFD study on mean flow engine for wind power exploitation. Energy Conversion and Management, 2011. 52(6): p. 2355-2359.

[5] Slaton, W.V., Zeegers, J.C.H., An aeroacoustically driven thermoacoustic heat pump. The Journal of the Acoustical Society of America, 2005. 117(6): p. 3628-3635.

[6] Slaton, W.V., Zeegers, J.C.H., Acoustic power measurements of a damped aeroacoustically driven resonator. The Journal of the Acoustical Society of America, 2005. 118(1): p. 83-91.

[7] Ullmann, A., The piezoelectric valve-less pump-performance enhancement analysis. Sensors and Actuators A: Physical, 1998. 69(1): p. 97-105.

[8] Ullmann, A., Fono, I., The piezoelectric valve-less pump-improved dynamic model. Journal of Microelectromechanical Systems, 2002. 11(6): p. 655-664.

[9] Lee, D.G., Or, S.W., Carman, G.P., Design of a piezoelectric-hydraulic pump with active valves. Journal of intelligent material systems and structures, 2004. 15(2): p. 107-115.

[10] Swift, G.W., Backhaus, S., A resonant, self-pumped, circulating thermoacoustic heat exchanger. Journal of the Acoustical Society of America, 2004. 116(5): p. 2923-2938.

[11] Gao, B., Wu, Z.H., Luo, E.C., Dai, W., Experimental demonstration of a novel heat exchange loop used for oscillating flow systems. Advances in Cryogenic Engineering, Vols 53a and 53b, 2008. 985: p. 1114-1121.

[12] Sun, D.M., Wang, K., Xu, Y., Shen, Q., Zhang, X.J., Qiu, L.M., Thermoacoustic compression based on alternating to direct gas flow conversion. Journal of Applied Physics, 2012. 111(9). 
[13] Sun, D.M., Wang, K., Qiu, L.M., Multistage thermoacoustic compression system. Chinese Patent, Patent No. 200810163521.3 .

[14] Sun, D.M., Wang, K., Lou, P., Zhao, Y.T., Qiu, L.M., A refrigeration system driven by thermoacoustic compressor. Chinese Patent, Application No. 201110261644.2.

[15] Backhaus, S.,Swift, G.W., A thermoacoustic Stirling heat engine. Nature, 1999. 399(6734): p. 335-338.

[16] Bisio, G.,Rubatto, G., Sondhauss and Rijke oscillations - thermodynamic analysis, possible applications and analogies. Energy, 1999. 24(2): p. 117-131.

[17] Chun, W., Oh, S.J., Lee, Y.J., Lim, S.H., Surathu, R., Chen, K., Acoustic waves generated by a TA (ThermoAcoustic) laser pair. Energy, 2012. 45(1): p. 541-545.

[18] Zhao, D., Ji, C., Li, S., Li, J., Thermodynamic measurement and analysis of dual-temperature thermoacoustic oscillations for energy harvesting application. Energy, 2014. 65(0): p. 517-526.

[19] Flitcroft, M., Symko, O.G., Ultrasonic thermoacoustic energy converter. Ultrasonics, 2013. 53(3): p. $672-676$.

[20] Roberts, D.C., Li, H., Steyn, J.L., Yaglioglu, O., Spearing, S.M., Schmidt, M.A., Hagood, N.W., A piezoelectric microvalve for compact high-frequency, high-differential pressure hydraulic micropumping systems. Journal of Microelectromechanical Systems, 2003. 12(1): p. 81-92.

[21] Park, J.M., Taylor, R.P., Evans, A.T., Brosten, T.R., Nellis, G.F., Klein, S.A., Feller, J.R., Salerno, L., Gianchandani, Y.B., A piezoelectric microvalve for cryogenic applications. Journal of Micromechanics and Microengineering, 2008. 18(1): p. 015023

[22] Gradin, H., Clausi, D., Braun, S., Stemme, G., Peirs, J., van der Wijngaart, W., Reynaerts, D., A low-power highflow shape memory alloy wire gas microvalve. Journal of Micromechanics and Microengineering, 2012. 22(7): p. 075002.

[23] Gedeon, D., Sage Stirling-Cycle Model Class Reference Guide. 5th ed. (Gedeon Associates, Athens, OH, 2007).

[24] Wollan, J.J., Swift, G.W., Backhaus, S.N., Gardner, D.L., Development of a thermoacoustic natural gas liquefier, in AIChE Meeting, March 11-14, 2002: New Orleans LA.

[25] Swift, G.W., Thermoacoustics: A unifying perspective for some engines and refrigerators. Acoustical Society of America, Sewickley, PA, 2002.

[26] Qiu, L.M., Lai, B.H., Zhao, Y.T., Sun, D.M., Zhang, X.J., Li Y.F., Study on the onset temperature of a standing-wave thermoacoustic engine based on circuit network theory. Science China Technological Sciences, 2012. 55(10): p. 2864-2868.

[27] Lai, B.H., Qiu, L.M., Li Y.F., Lou, P., Sun, D.M., Simulation of onset process of standing wave thermoacoustic engine based on thermoacoustic network theory. Journal of Zhejiang University (Engineering Science), 2011. 45(6): p. 1130-1135. (in Chinese)

[28] Sun, D.M., Qiu, L.M., Wang, B., Xiao, Y., Transmission characteristics of acoustic amplifier in thermoacoustic engine. Energy Conversion and Management, 2008. 49(5): p. 913-918.

[29] Sun, D.M., Qiu, L.M., Wang, B., Xiao, Y., Novel Helmholtz resonator used to focus acoustic energy of thermoacoustic engine. Applied Thermal Engineering, 2009. 29(5-6): p. 945-949.

[30] Gillman, B.J., A Study of coupled thermoacoustic engines. The University of Utah, 2009. Doctor of Philosophy.

[31] Sun, D.M., Qiu, L.M., Zhang, W., Yan, W.L., Chen, G.B., Investigation on traveling wave thermoacoustic heat engine with high pressure amplitude. Energy Conversion and Management, 2005. 46(2): p. 281-291. 
[32] Yu, G.Y., Luo, E.C., Dai, W., Wu, Z.H., An energy-focused thermoacoustic-Stirling heat engine reaching a high pressure ratio above 1.40. Cryogenics, 2007. 47(2): p. 132-134. 